\title{
Modelo de Sistema de Gestión de Calidad para la Puesta en Marcha de Cursos no Presenciales: Instrumentos de Seguimiento y Evaluación
}

\author{
Myriam G. Llarena, Silvia L. Villodre, Francisco A. Pontoriero y Adela B. Cattapan \\ Facultad de Ciencias Exactas, Físicas y Naturales. Universidad Nacional de San Juan, \\ Av. Ignacio de la Roza y Meglioli. Rivadavia, CP.5423 San Juan-Argentina. \\ (e-mail: myriam.llarena@gmail.com; svillodre@gmail.com; ruffopontoriero@gmail.com; \\ acatapan@gmail.com)
}

Recibido May. 23, 2014; Aceptado Ago. 01, 2014; Versión final recibida Sep. 29, 2014

\begin{abstract}
Resumen
El propósito de este trabajo es responder a interrogantes que se plantean cuando una institución educativa inicia la tarea de construir un sistema de gestión de calidad, que contemple aspectos inherentes a la gestión de los procesos de planificación, implementación y ejecución de cursos no presenciales. La primera etapa de la investigación consistió en un análisis documental, descriptivo-comparativo, de estándares de calidad vigentes. En la segunda, se propuso un modelo del sistema, que define los procesos a tener en cuenta en la implementación de cursos, así como estrategias para su seguimiento y evaluación. Dada su importancia, los instrumentos generados para evaluar el cumplimiento de indicadores de procesos son detallados para el proceso Ejecución del Proyecto Educativo. Actualmente las estrategias e instrumentos desarrollados en el estudio están siendo implementadas en cursos de la Facultad de Ciencias Exactas de la Universidad Nacional de San Juan en Argentina.
\end{abstract}

Palabras clave: modalidad no presencial, sistema de gestión de calidad, seguimiento y evaluación, estándares de calidad

\section{Quality Management System Model for the Implementation of Distance Courses: Monitoring Tools and Evaluation}

\begin{abstract}
The purpose of this work is to answer questions that arise when an educational institution begins the task of building a quality management system, which covers aspects relating to the management of the planning, implementation and execution of distance learning. The first stage of the research consisted of a documentary, descriptive and comparative analysis of current quality standards. In the second stage, a model of the system was proposed, defining the processes to be considered in the implementation of courses, as well as strategies for monitoring and evaluation. Because of their importance, the instruments created to evaluate compliance of the process indicators are detailed for the Education Project Implementation. A present, the strategies and tools developed in the study are being implemented in courses of the Faculty of Exact Sciences of the National University of San Juan in Argentina.
\end{abstract}

Keywords: online learning approach, quality management system, monitoring and evaluation, quality standards 


\section{INTRODUCCIÓN}

En el año 2004 el Ministerio de Cultura y Educación Argentino, a través de la Resolución № 1717/ MCE regula las actividades referidas a la modalidad a distancia, y expresa en su artículo $5^{\circ}$, título II: "La presentación de una carrera de pregrado, grado o posgrado tendrá la estructura de un proyecto académico, que incluya en sus diferentes componentes el sistema de evaluación interna del mismo así como propuestas de mejora continua". La Facultad de Ciencias Exactas Físicas y Naturales (FCEFyN) crea en el año 2005 el Programa Permanente de Investigación en Educación a Distancia-EaD- desde el cual se realiza oferta académica con Tecnologías de Información y Comunicación (TIC), que incluye cursos de posgrado, apoyo a la modalidad presencial de cátedras de grado y el curso de ingreso no presencial a las carreras de la mencionada Facultad. La Universidad de San Juan (UNSJ), en el año 2010 reglamenta las actividades académicas en la modalidad a distancia, y especifica "En la Modalidad Educación a Distancia se requiere de un Sistema de Gestión, que garantice la calidad de la oferta académica". En función de este requerimiento, se ha desarrollado en el marco del Programa EaD un modelo de Sistema de Gestión de Calidad (SGC), para prestar el servicio de educación no presencial ajustado a estándares de calidad vigentes en esta modalidad, que a su vez sean contextualizados a la realidad de la UNSJ.

Para la implementación del modelo de SGC se plantearon las siguientes cuestiones:¿Cómo lograr que los procesos inherentes a la modalidad de Educación no Presencial se desarrollen en un marco de calidad? ¿Cuáles son los mecanismos más adecuados para garantizar que la organización del sistema sea pertinente y pueda ser evaluada sistémicamente? ¿Cómo articular las acciones de equipos diferentes de trabajo, para que la interacción entre ellos resulte fluida? ¿Qué mecanismos se pueden articular para asegurar la retroalimentación que favorezca procesos de mejora continua?

La determinación de un SGC para la gestión, seguimiento, evaluación y mejora de los procesos inherentes a cursos no presenciales, debe estar sustentada en una concepción de calidad. En las memorias del Proyecto Centro Virtual para el desarrollo de Estándares de Calidad para la Educación Superior a Distancia del Instituto Latinoamericano y del Caribe de Calidad en Educación Superior a Distancia (CALED), uno de los referentes manifiesta que durante muchos años la calidad en la Educación Superior fue vista como algo deseable pero de definición eludible, misteriosa y subjetiva. Sin embargo, en la última década, diversos congresos internacionales sobre universidades, tanto presenciales como a distancia, y especialmente la UNESCO, le asignaron extraordinaria importancia a la Calidad. Casas, considera necesario que se desmitifiquen las características de la Calidad y se precisen sus parámetros, y especialmente los estándares, criterios e indicadores (Rubio, 2010).

El programa de investigación de la Facultad de Ciencias Exactas ancla en el concepto de calidad propuesto desde la Calidad Total. La Calidad Total es una filosofía básica de administración propuesta por Deming, compatible con los métodos estadísticos que utilizaba desde la década del 40 para la evaluación de la calidad. Al observar el fracaso de estos métodos, Deming reflexiona acerca de la causa y para evitarlo paulatinamente llega a la conclusión que se necesita una filosofía aplicada tanto a productos como a servicios, ésta se conoce como los catorce puntos, y constituyen la columna vertebral de la Calidad Total (Deming,1986). Los siguientes son los principios que pueden derivarse de los catorce puntos, los cuales resultan orientadores a este trabajo, adecuando la terminología al contexto educativo:: i) Mejora permanente de todo lo que la institución pretende alcanzar, sobre la base de objetivos claros y explícitos; ii) Proceso orientado a la satisfacción de expectativas y necesidades de sus destinatarios, usuarios/alumnos; iii) Garantía de la calidad de los procesos internos como medida para alcanzar la calidad del servicio; iv) Trabajo en equipo; v) Formación de las personas implicadas; y vi) Prevención en lugar de la supervisión y detección de errores (Deming, 1986).

La adecuación de la terminología utilizada por Deming al contexto educativo, consiste en el uso de los vocablos institución u organización que ofrece el servicio educativo en lugar de empresa, usuarios, alumnos o participantes en lugar de clientes y curso en lugar de producto.

Expertos en Calidad Total, refieren a calidad: "Conformidad con los requerimientos" dice Crosby (1987) ó "adecuado para el uso" Juran y Gryna (1993). Un sistema eficaz para integrar los esfuerzos en materia de desarrollo de calidad, mantenimiento y mejoramiento de calidad, realizados por los diversos grupos de una organización de modo que sea posible producir bienes y servicios a los niveles más económicos y que sean compatibles con la satisfacción de los clientes (Feigenbaum ,1963). La primera definición centra su atención en el usuario, la segunda en el producto o servicio en sí. La calidad total requiere de ambos requisitos, calidad del servicio y satisfacción del usuario.

La calidad debe ser definida y medida si se quiere mejorar, "No se puede mejorar lo que no se puede medir" (De Marco, 1986). El especialista del CALED Luis Romero expresa, en las memorias del Proyecto Centro 
Virtual para el desarrollo de Estándares de Calidad para la Educación Superior a Distancia (2010), que la calidad es un tema que ha adquirido especial vigencia a partir de los modelos aplicados a la empresa; pero, en realidad, ha sido el tema de siempre de las universidades desde su origen. Sin embargo, la amplitud semántica del término calidad encierra el peligro de considerarlo de forma ambigua y laxa, donde todo cabe. En este sentido los modelos empresariales aportaron una forma de hacer operativa la calidad a través de instrumentos de medida más o menos objetivos, que son cada vez más aceptados y utilizados en educación. Sin embargo estos modelos no se pueden considerar como recetas infalibles. La norma ISO define Calidad como el grado en que un conjunto de características inherentes (a un objeto, producto, servicio, etc.) cumple con una necesidad o expectativa establecida, generalmente implícita u obligatoria (ISO 9000:2000, 2005).

El desafío de las universidades es entonces definir la calidad deseada e identificar técnicas para medirla. Se debe atender no sólo la calidad del proceso sino también la satisfacción del usuario; conocer lo que él percibe permitirá a la institución que ofrece el servicio, tomar decisiones tendientes a satisfacer sus necesidades y exigencias (LLarena, 2008). La filosofía de la calidad total se basa en la gestión y control de la calidad en todas y cada una de las componentes del sistema y durante todo el proceso (Deming,1986).Gestionar la calidad implica determinar y aplicar políticas de calidad. No es fácil establecer el conjunto de propiedades o características que determinen que un servicio es de calidad. Para ayudar a resolver esta problemática existen los Modelos de Calidad, que permiten descomponer un concepto en forma jerárquica en subconceptos que facilitan la evaluación de su calidad. La calidad se convierte de esta manera en algo concreto, que se puede definir, planificar, evaluar y por tanto mejorar.

Determinar un modelo consiste en especificar las dimensiones, así como los estándares e indicadores que servirán para medir el cumplimiento de los objetivos de calidad propuestos. Las dimensiones son aquellos factores que se consideran fundamentales para la calidad del servicio (Rubio, 2010). Expresa Deming (1986) que existen reglamentos establecidos por el gobierno, estándares voluntarios establecidos por comités y también orientaciones dadas por empresas e individuos. La conformidad sólo se puede juzgar en función de un ensayo y de un criterio, que deben estar definidos en términos estadísticos para tener sentido. Un grupo de expertos del CALED expresan la falta de uniformidad en la definición de estándar en la literatura revisada y adoptan la siguiente definición, a la que se adhiere en este trabajo: "Nivel o grado definido como necesario e indispensable para que algo pueda considerarse aceptable" (Díaz, 2001). Ese nivel o grado se refiere a la realización de un conjunto de actividades.

Respecto al concepto de Indicador, referentes de la UNESCO señalan que los indicadores educativos son: "Un artificio que proporciona información relevante acerca de algún aspecto significativo de la realidad educativa, lo más habitual es que dicho artificio consista en algún tipo de dato de carácter cuantitativo generalmente una medida estadística" (Tiana,1997). Para definir un modelo contextualizado a la realidad institucional de la UNSJ, pero que a la vez atendiera estándares vigentes, se compararon estándares vinculados con el e-learning. Así el modelo que se propone está sustentado en las normas ISO (Internacional Standards Organization): 9000:2000, el modelo EFQM (European Foundation for Quality Management) de excelencia empresarial y las Buenas Prácticas realizadas por instituciones líderes en la temática o Sistemas Benchmarking.

Expresa Aja (2002) que los exámenes para determinar la calidad de los productos y servicios son el resultado del desarrollo de normas internacionales, regionales y nacionales dirigidas a auxiliar a las partes interesadas: proveedores, productores, comercializadores y clientes en el hallazgo de un instrumento de consenso común para la evaluación. Algunos de los estándares más conocidos son las normas ISO de las series 9000 y 9001 . Las normas ISO 9000:2000 son documentos de carácter técnico desarrollados para servir de referente a toda organización interesada en la implementación y operación efectiva de un SGC. Esta norma especifica que: Sistema de Gestión es un "Sistema para establecer la política y los objetivos, y para lograr dichos objetivos" (ISO, 2008).

Entre sus principios se destacan: enfoque orientado al cliente, basado en procesos, orientado a la mejora continua (ciclo de Deming: planear, hacer, verificar, actuar) y basado en hechos para toma de decisiones. La norma ISO 9000 no establece los requisitos que deben cumplirse para la prestación de los servicios, no especifica cómo debe ser el servicio, qué características y rendimientos preestablecidos deben alcanzarse. La norma exige que los requisitos deban ser identificados y documentados así como cualquier modificación que se produzca en los mismos. Son las organizaciones quienes deben proporcionar sistemáticamente servicios que reúnan las condiciones acordadas, y el cumplimiento de los requisitos pactados es lo que determina la conformidad o no del servicio (ISO, 2008).

El sistema de gestión de calidad contempla todos los aspectos que van desde el diseño del servicio hasta su puesta a disposición de los usuarios. La norma ISO 9001 especifica los requisitos genéricos que debe 
reunir el SGC, aplicables a toda clase de organizaciones con independencia de la naturaleza de los productos o servicios que presten. Propone un modelo de procesos orientado al cliente/usuario, quién determina los requisitos de entrada del SGC, poniendo énfasis en el seguimiento y medición de la información relacionada con la percepción del cliente acerca de cómo la organización satisface sus requerimientos. Refiere a Proceso como conjunto de actividades mutuamente relacionadas o que interactúan, las cuales transforman elementos de entrada en resultados (ISO 9000:2000, 2005).

Deming (1986) considera que si no se puede describir lo que se está haciendo como un proceso, realmente no es posible saber lo que se está haciendo y para ello demostró que la producción es un sistema y no una secuencia de procesos mecánicos sin relación. Debido a lo anterior, el desempeño de una organización puede mejorarse por medio de la aplicación y el uso del enfoque basado en procesos, donde estos últimos se gestionan como un sistema gracias a la creación y entendimiento de una red de procesos y sus interacciones (Hernández et al.,2013).

El apartado 4.1 de la norma ISO 9000:2000, propone una serie de pasos para el establecimiento, implantación y mantenimiento de un SGC (Tabla 1).

Tabla 1: Requisitos Apartado 4.1- Norma 9000:2000- Enfoque por procesos

\begin{tabular}{|l|l|}
\hline Pasos del enfoque por Procesos & Requisitos Apartado 4.1-Norma 9000:2000 \\
\hline $\begin{array}{l}\text { 1-Identificar y determinar secuencia } \\
\text { de Procesos }\end{array}$ & $\begin{array}{l}\text { a) Identificar los procesos necesarios para el SGC. } \\
\text { b) Determinar la secuencia e interacción de estos procesos. }\end{array}$ \\
\hline 2-Descripción de los Procesos & $\begin{array}{l}\text { c) Determinar los criterios y métodos necesarios para } \\
\text { asegurarse que tanto la operación como el control de los } \\
\text { procesos sean eficaces. }\end{array}$ \\
\hline $\begin{array}{l}\text { 3- Seguimiento y medición de los } \\
\text { procesos }\end{array}$ & $\begin{array}{l}\text { d) Asegurar la disponibilidad de recursos e información } \\
\text { necesarios para apoyar la operación y el seguimiento de los } \\
\text { procesos. } \\
\text { e) Realizar el seguimiento, medición y análisis de los procesos. }\end{array}$ \\
\hline 4- Mejora de los procesos & $\begin{array}{l}\text { f) Implementar las acciones necesarias para alcanzar los } \\
\text { resultados planificados y la mejora continua de los procesos. }\end{array}$ \\
\hline
\end{tabular}

La European Foundation for Quality Management (EFQM), propone un modelo de gestión de calidad total, que ayuda a las organizaciones a establecer un sistema de gestión, midiendo el punto en que se encuentran dentro del camino hacia la excelencia. El modelo analiza cómo la organización combina factores y estrategias a través de sus procesos, con el objeto de producir ciertos resultados. Refiere como resultados los aspectos relativos a lo que la organización ha conseguido y está consiguiendo y como agente a los aspectos relativos a cómo se obtienen esos resultados. Este modelo está orientado hacia los resultados, teniendo en cuenta la satisfacción de todas las partes interesadas y considera que la gestión por procesos y hechos, permite a las organizaciones actuar de manera mas efectiva: i) Considera que un Sistema de Gestión de Calidad es un "Esquema general de procesos y procedimientos que se emplea para garantizar que la organización realiza todas las tareas necesarias para alcanzar esos objetivos"; ii) Define proceso como una secuencia de actividades que van añadiendo valor mientras se produce un determinado producto o servicio a partir de determinadas aportaciones. Denomina "valor agregado a la diferencia que aporta un proceso entre la entrada que recibe y la salida que entrega; iii) Clasifica los procesos en Procesos Estratégicos, Claves y de Soporte (González, 2011).

Los Procesos estratégicos son aquellos que proporcionan directrices a todos los demás procesos. Suelen referir a las leyes, normativas aplicables al servicio y son realizados por la dirección (González, 2011). Están asociados al rumbo de la organización a mediano y largo plazo, misión-visión (Hernández et al, 2013). En función del valor agregado que aportan al usuario, los procesos se clasifican en procesos clave y procesos de soporte: Procesos clave, aportan valor agregado directamente al usuario, son aquellos por los que el usuario solicita el servicio y los que percibe directamente. Procesos soporte, aportan valor agregado a otros procesos. Forman la infraestructura organizativa necesaria para los procesos clave (González, 2011).

Los sistemas basados en la práctica del benchmarking consideran que la calidad se logra a partir de la observación y comparación con centros referentes en excelencia. De esta manera es posible definir herramientas e indicadores que permiten observar, medir y comparar con las buenas prácticas que ellos realizan y/o proponen. Expresa González (2011) : Entendemos por buena práctica una forma de realizar, organizar y sistematizar un proceso de trabajo relacionado con generar, desarrollar, difundir y ejecutar una actividad e-learning, que potencie la obtención de resultados de calidad, fundamentalmente desde la 
perspectiva del aprendizaje que logran las personas...En este sentido, la detección de buenas prácticas tiene como propósito ponerlas a disposición de las instituciones que planean ofrecer actividades e-learning, de manera de proporcionarles un conjunto de orientaciones que les permitan examinar el diseño, desarrollo e implementación de sus actividades de capacitación.

Hernández et al.(2013) manifiestan como un hallazgo relevante de los resultados de la investigación realizada para conocer la situación de 21 sistemas de gestión de calidad implementados en la Universidad Autónoma de Baja California, la ausencia de los procesos relacionados con las actividades académicas.

La particularidad del presente trabajo consiste no sólo en la propuesta de un enfoque global, donde se consideran todos los procesos involucrados en la puesta en marcha de un Proyecto Educativo (PE) en entornos virtuales, sino también en que se muestran instrumentos para evaluar la calidad de los mismos. Se describe la herramienta utilizada para la implementación del Plan de Calidad propuesto, así como los instrumentos puestos a disposición del alumno para evaluar el cumplimento de sus expectativas y el grado de satisfacción respecto de las instancias previstas para el desarrollo del proceso de Enseñanza/Aprendizaje.

\section{MODELO DE SISTEMA DE GESTIÓN E INSTRUMENTOS DE EVALUACIÓN}

La puesta en marcha de un Proyecto Educativo (PE) no presencial involucra su planificación, diseño, desarrollo, implementación en un entorno virtual y ejecución. Esto requiere la realización de procesos complejos, que deben ser planificados, gestionados y evaluados para asegurar la calidad y pertinencia de la oferta académica en esa modalidad. Para gestionar y garantizar la calidad se necesita un SGC que determine los procedimientos y responsabilidades necesarios para que una organización proporcione confianza del cumplimiento de los requisitos de calidad pertinentes para lograr sus objetivos. El siguiente gráfico resume las características de un SGC:

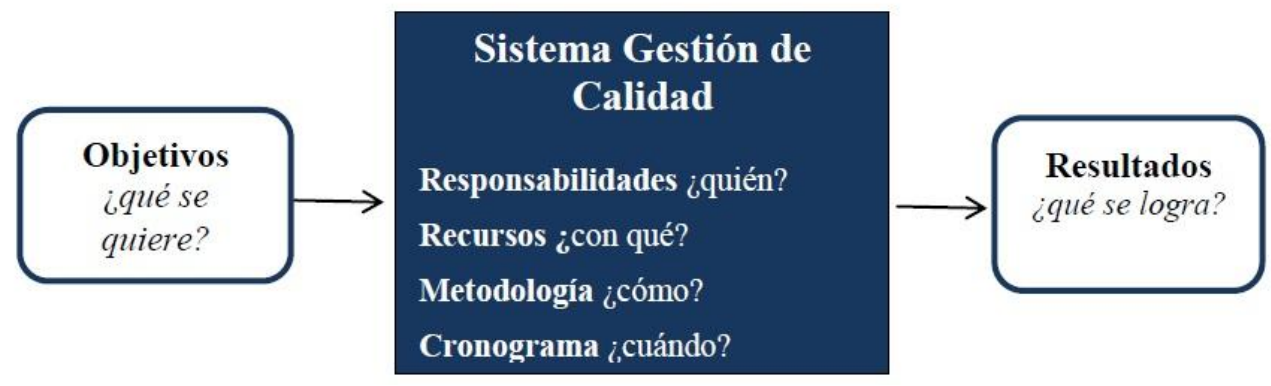

Fig. 1: Sistema de gestión como herramienta para alcanzar los objetivos

A continuación se describe cómo se atendieron los cuatro requisitos del apartado 4.1 de la norma ISO 9000:2000, referidos a los procesos: a) Identificación y secuenciación de proceso; b) Descripción de los procesos; c) Seguimiento y medición de los procesos; y d) Mejora de los procesos

\section{a- Identificación y secuenciación de procesos}

Moneta, et al. (2013) expresan que todo PE puede pensarse como un mapa de procesos estratégicos, claves y de apoyo, cada uno de los cuales tiene como insumos las necesidades de determinados grupos de interés (alumnos, docentes, equipos de producción, directivos, entre otros) y como salida los resultados requeridos que satisfarán las demandas de los grupos destinatarios. Los procesos inherentes a la puesta en marcha de un curso en la modalidad no presencial, se sistematizaron en procesos estratégicos, claves y de soporte, como se muestra en la figura 2. Proceso de Soporte, es el proceso administrativo, que consiste en la gestión administrativa de los distintos procesos.

En los Procesos Estratégicos se determinan objetivos, normativas y lineamientos de gestión, didácticos y tecnológicos. Los procesos clave del proyecto educativo (PE), son cinco: 1. Gestionar alta del PE; 2. Diseñar y desarrollar didácticamente el PE; 3. Implementar el PE; 4. Ejecutar el PE y 5. Seguir y evaluar el PE.

1. Gestionar alta del PE: En el que se analiza la factibilidad macro del proyecto entrante y en caso afirmativo se realiza la planificación de dicho proyecto. La salida de este proceso es el Plan de Calidad.

2. Diseñar y desarrollar didácticamente el PE: Se trata del diseño de toda la propuesta, se centra específicamente en la planificación del proceso de enseñanza- aprendizaje, atendiendo las necesidades de 
usuarios y el contexto donde se desarrollará. Se definen objetivos, seleccionan contenidos, proponen actividades, se diseña la evaluación y se eligen los recursos más adecuados para los procesos de aprendizaje. La salida de este proceso es el prototipo del aula virtual (AV) en un espacio de prueba, de acuerdo al diseño didáctico, para verificar el cumplimiento de normativas y funcionalidades establecidas.

3. Implementar el PE: Consiste en la habilitación del aula virtual y el alta de alumnos.

4. Ejecutar el PE: Consiste en el desarrollo del curso, durante el cual se debe realizar el seguimiento, evaluación y ajustes pertinentes.

5. Seguir y evaluar el PE: se ejecuta en todos los procesos y consta de los subprocesos:

5.1 Diseñar la Evaluación, en el que se confeccionan los instrumentos para la evaluación de cada proceso.

5.2 Evaluar procesos.

5.3 Proponer reajustes y/o mejoras.

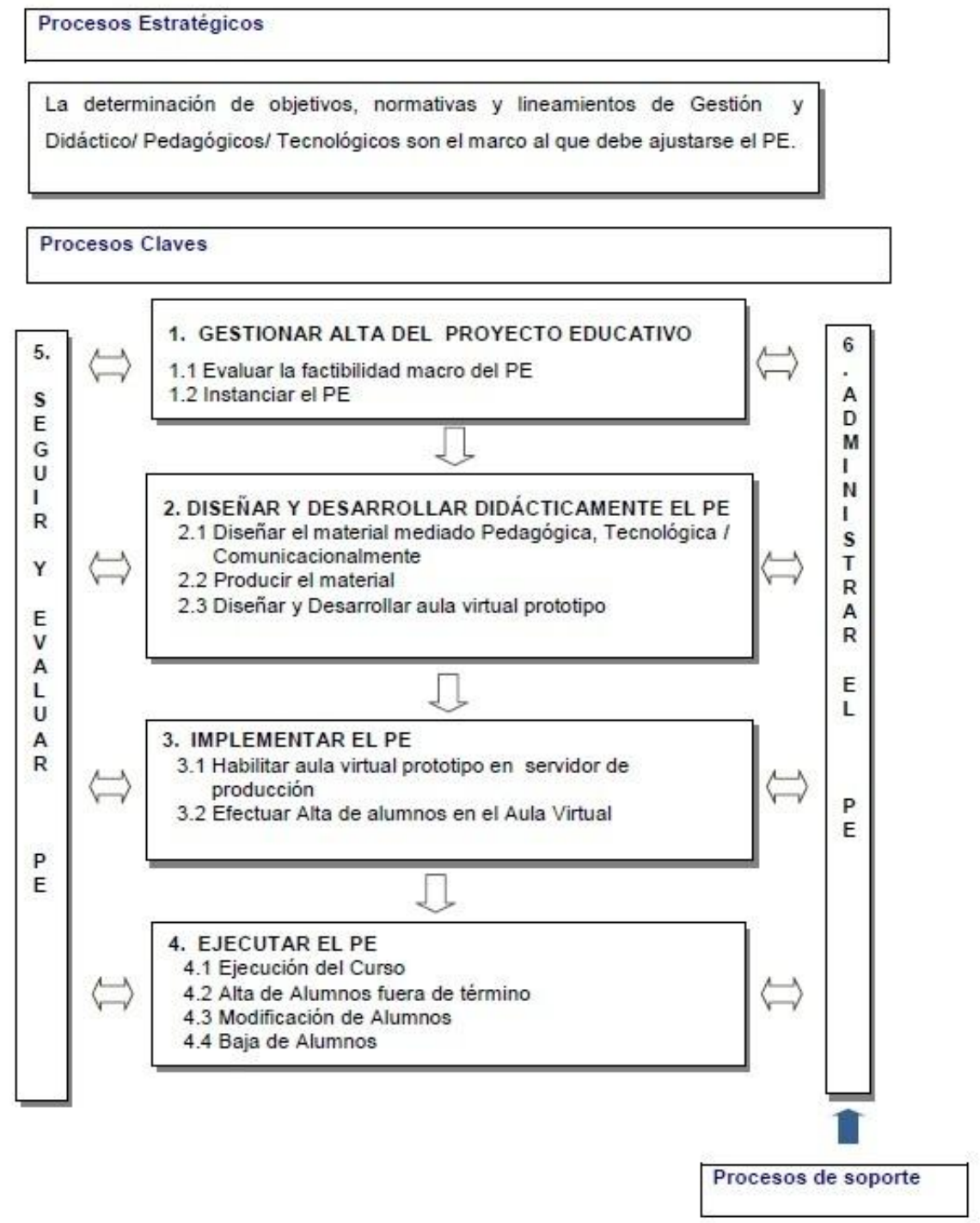

Fig 2. Mapa de Procesos para Cursos Virtuales

EI SGC queda materializado a través del Plan de Calidad elaborado para cada uno de los procesos. Plan de Calidad es el documento que especifica cómo se aplicarán los procedimientos y los recursos (ISO 9000:2000). Documentar el Plan de Calidad de un curso, en el que se especifica cómo se realizarán los procedimientos así como los recursos y tiempos asignados para ello, es prácticamente inabordable sin la ayuda de herramientas de Planificación y Gestión de Proyectos. Para este proceso, se utilizó la herramienta de software libre y código abierto dotProyect, que permite al responsable del seguimiento de cada proceso conocer el estado de las actividades que lo constituyen, como se muestra en la siguiente tabla. 
Tabla 2: Plan de Calidad de un Curso Virtual-FCEFN

\begin{tabular}{|c|c|c|}
\hline Nombre de la Tarea & Usuarios Asignados & Fecha Inicio \\
\hline 1. GESTIONAR ALTA PE & adeluca $(100 \%)$ & 01/09/2014 02:45 pm \\
\hline 1.1. Evaluar la factibilidad macro del PE & adeluca $(40 \%)(+2)$ & 01/09/2014 03:45 pm \\
\hline $\begin{array}{l}\text { 1.1.2 Cargar información del curso en Registro de Cursos de } \\
\text { la Unidad }\end{array}$ & adominguez (100\%) & 01/09/2014 04:00 pm \\
\hline $\begin{array}{l}\text { 1.1.3 Entregar formulario de no aceptación del curso } \\
\text { discriminando causas inherentes al solicitante }\end{array}$ & adominguez $(50 \%)(+2)$ & 01/09/2014 04:15 pm \\
\hline $\begin{array}{l}\text { 1.1.1 Verificar estado y archivar Formulario de solicitud } \\
\text { implementación aula virtual }\end{array}$ & rposito $(40 \%)(+2)$ & 01/09/2014 09:00 pm \\
\hline 1.2 Instanciar el PE & rposito $(70 \%)(+1)$ & 08/09/2014 08:00 am \\
\hline 1.2.1 Elaborar Plan de Calidad del curso & adominguez (100\%) & 03/09/2014 04:15 pm \\
\hline 1.2.2 Elaborar Diagrama de actividad(Gantt) & rposito $(100 \%)$ & 03/09/2014 04:15 pm \\
\hline $\begin{array}{l}\text { 1.2.3 Solicitud de habilitación del aula virtual prototipo en } \\
\text { servidor de desarrollo y usuarios editores }\end{array}$ & rposito $(100 \%)$ & 03/09/2014 04:30 pm \\
\hline $\begin{array}{l}\text { 1.2.4 Habilitación del aula virtual prototipo en servidor de } \\
\text { desarrollo y usuarios editores }\end{array}$ & rogeliofullana (100\%) & 08/09/2014 08:00 am \\
\hline $\begin{array}{l}\text { 2. DISEÑAR Y DESARROLLAR DIDÁCTICAMENTE EL } \\
\text { PROYECTO EDUCATIVO }\end{array}$ & adeluca $(100 \%)$ & 08/09/2014 08:00 am \\
\hline $\begin{array}{l}\text { 2.1 Diseñar el material mediado Pedagógica, Tecnológica- } \\
\text { Comunicacionalmente }\end{array}$ & rposito (100\%) & 08/09/2014 08:00 am \\
\hline $\begin{array}{l}\text { 2.1.1 Habilitación como usuario del espacio de la comunidad } \\
\text { EAD para extracción/consulta }\end{array}$ & rposito $(100 \%)$ & 08/09/2014 08:00 am \\
\hline $\begin{array}{l}\text { 2.1.2 Informar al docente sobre los responsables técnico, } \\
\text { pedagógico y de reglamentaciones vigentes para el diseño } \\
\text { de materiales }\end{array}$ & rposito $(100 \%)$ & 08/09/2014 08:00 am \\
\hline $\begin{array}{l}\text { 2.1.3 Revisión del cumplimiento de las actividades previstas } \\
\text { en el formulario acuerdo }\end{array}$ & rposito $(100 \%)$ & 08/09/2014 08:00 am \\
\hline 2.2 Producir el material & adominguez (100\%) & 09/09/2014 08:00 am \\
\hline $\begin{array}{l}\text { 2.2.1 Comunicar al docente la disponibilidad de instrumen- } \\
\text { tos para controlar cumplimiento de lineamientos pedagógi- } \\
\text { cos, tecnológicos, reglamentaciones y disposiciones vigentes }\end{array}$ & adominguez (100\%) & 09/09/2014 01:00 pm \\
\hline $\begin{array}{l}\text { 2.2.2 Evaluar el cumplimiento de cronograma de desarrollo } \\
\text { del material mediado a cargo de la unidad especializada }\end{array}$ & adominguez (100\%) & 09/09/2014 01:00 pm \\
\hline 2.3. Diseñar y Desarrollar el aula virtual prototipo & adominguez (40\%) (+2) & $15 / 09 / 201408: 00 \mathrm{am}$ \\
\hline 2.3.1 Subir el material mediado & adominguez (100\%) & $10 / 09 / 201401: 30 \mathrm{pm}$ \\
\hline $\begin{array}{l}\text { 2.3.2 Controlar si el diseño editado cumple con todas las } \\
\text { especificaciones preestablecidas }\end{array}$ & adominguez $(50 \%)(+1)$ & 11/09/2014 08:30 am \\
\hline $\begin{array}{l}\text { 2.3.3 Evaluar el cumplimiento de lineamientos pedagógicos } \\
\text { y tecnológicos con un instrumento adecuado }\end{array}$ & mllarena $(100 \%)$ & 15/09/2014 08:00 am \\
\hline $\begin{array}{l}\text { 2.3.4 Evaluar el cumplimiento de reglamentaciones y } \\
\text { aspectos legales vigentes con un instrumento adecuado }\end{array}$ & mllarena $(100 \%)$ & $15 / 09 / 201408: 00 \mathrm{am}$ \\
\hline 2.3.5 Informar el resultado del control al editor & rposito $(100 \%)$ & $15 / 09 / 201408: 00 \mathrm{am}$ \\
\hline $\begin{array}{l}\text { 2.3.6 Avisar al campus para la publicación del aula en el } \\
\text { servidor de producción }\end{array}$ & adominguez (100\%) & 15/09/2014 08:00 am \\
\hline 3. IMPLEMENTAR PROYECTO EDUCATIVO & $\operatorname{admin}(100 \%)$ & $15 / 09 / 201408: 00 \mathrm{am}$ \\
\hline 3.1 Habilitar el aula virtual en el servidor de producción & adominguez $(50 \%)(+1)$ & 15/09/2014 08:00 am \\
\hline $\begin{array}{l}\text { 3.1.1 Publicación del aula virtual prototipo en el servidor de } \\
\text { producción }\end{array}$ & rogeliofullana (100\%) & 15/09/2014 08:00 am \\
\hline 3.1.2 Verificación /Testeo de funcionalidades & adominguez (100\%) & $15 / 09 / 201408: 00 \mathrm{am}$ \\
\hline $\begin{array}{l}\text { 3.1.3 Habilitación en el Campus Virtual de la página para } \\
\text { inscripción de alumnos }\end{array}$ & rogeliofullana $(100 \%)$ & 15/09/2014 08:00 am \\
\hline $\begin{array}{l}\text { 3.1.4 Comunicación al alumno el modo de ingreso a la } \\
\text { página de inscripción }\end{array}$ & adominguez (100\%) & 15/09/2014 10:00 am \\
\hline 3.2. Alta de alumnos del aula virtual & adominguez (40\%) (+2) & $15 / 09 / 201401: 00 \mathrm{pm}$ \\
\hline 4. EJECUTAR EL PE & adominguez $(40 \%)(+2)$ & 22/09/2014 08:00 am \\
\hline 6. SEGUIR Y EVALUAR EL PROYECTO EDUCATIVO & mllarena $(50 \%)(+3)$ & 22/09/2014 08:00 am \\
\hline
\end{tabular}


Los diferentes colores que se observan en la última columna de la tabla 2, corresponden a un código que permite al responsable del seguimiento de los procesos, identificar las tareas que comenzaron a tiempo, las que debieron comenzar y aún no lo hicieron, las atrasadas y las que ya finalizaron, es decir le permite determinar el estado de los proyectos así como la eficiencia de los agentes afectados (Ronald y Rodríguez, 2007).

\section{b. Descripción de los Procesos}

González (2011) sugiere los siguientes aspectos a tener en cuenta para describir un proceso: i) Definirlo, esto es especificar de qué se trata y sus límites. Determinar cuál es su misión y objetivos. Identificar quién es el beneficiario (cliente) del proceso así como identificar los estándares de calidad aceptables para él; ii) Especificar Entradas (elementos necesarios) y Salidas (resultados obtenidos) del proceso; iii) Detallar Recursos(elementos fijos necesarios) y Procedimientos (reglas e instrucciones que determinan la manera de proceder o de actuar); iv) Establecer quién es el propietario o responsable del proceso; y v) Especificar el método de evaluación y de revisión que se adoptará para introducir mejoras en el proceso, lo que incluye determinar indicadores del proceso.

Cuando referimos a indicadores de proceso, se alude a estándares, esto es afirmaciones sobre el nivel y calidad aceptable en la dimensión evaluada. Los indicadores del proceso Ejecución del Curso, se seleccionaron atendiendo la importancia que en él adquieren la interactividad e interacción. Entendiendo por interactividad la relación del alumno con el material de aprendizaje y la tecnología; y por Interacción la relación entre los actores del proceso: alumno/docente y alumnos entre sí. A fin de documentar los procesos, tal como lo exige la norma ISO y atendiendo los aspectos a tener en cuenta para su descripción, sugeridos por González, se ha confeccionado una Plantilla para documentar procesos. La tabla 3 muestra dicho instrumento, ejemplificado en el proceso Ejecución del Curso.

Tabla 3: Plantilla para documentar procesos

\begin{tabular}{|l|l|l|}
\hline \multicolumn{2}{|l|}{ Plantilla para documentar procesos } \\
\hline Ejecución del curso & Clave: 4.1 & Responsable del proceso: Interesado/Docente \\
\hline Año de inicio de operación: 2013 & No. de revisión: 1 &
\end{tabular}

a) Objetivo del proceso/ sistema:El propósito de este proceso es brindar los recursos necesarios para que el usuario alumno transite sin dificultadlas instancias previstas para el proceso de Enseñanza/Aprendizaje.

b) Requerimientos de los "clientes" Alumnos: Contar con los recursos necesarios para lograr los objetivos propuestos en el curso. Cumplir sus expectativas.

Descripción del proceso

c) Límites del proceso. ¿Cuándo se inicia y cuando termina el proceso?

El proceso comienza cuando el Interesado/Docente da la bienvenida a los alumnos del curso, indica pautas generales del mismo, y los invita a recorrer las distintas secciones del aula virtual.

El proceso finaliza cuando se ha cumplido la planificación y el cronograma previsto para el curso.

d) ¿Cuáles son los insumos (ingresos) que inician el proceso y quiénes son sus proveedores?

Entrada: PE implementado como aula virtual. Los proveedores son el Docente/ Interesado, Coordinador académico , Responsable de Soporte Tecnológico y Responsable del Campus Virtual

e) ¿Quiénes son los "clientes" del proceso?:Alumnos/Usuarios.

f) Salidas o resultados del proceso: Salida: PE ejecutado.

g) Descripción: El proceso comienza cuando el Interesado/Docente da la bienvenida a los alumnos del curso, indica pautas generales del mismo, cronograma, metodología de trabajo, entre otros y los invita a recorrer las distintas secciones del aula virtual. El docente debe participar activamente acompañando al alumno de manera tal que logre cumplir los objetivos del curso, así como las expectativas de sus alumnos, propiciando su aprendizaje autónomo y el trabajo colaborativo con sus pares. Si el docente lo requiere contará con el asesoramiento del Coordinador académico y el responsable de Soporte Tecnológico. El proceso finaliza cuando se ha cumplido la planificación y el cronograma previsto para el curso.

h) Interacción con otros procesos / sistemas

\begin{tabular}{|l|l|}
\hline Proceso/Sistema & Interacción \\
\hline Implementar el Proyecto Educativo & Relación temporal ya que para desarrollar el curso, el PE debe estar \\
\hline
\end{tabular}
implementado y habilitado en la plataforma. 
Tabla 3 (continuación)

Indicadores de resultado

1. El aula virtual posee una sección de Información General, que permite al alumno conocer aspectos esenciales para el desarrollo del curso.

2. Existe una sección que facilita al alumno la exploración y adaptación al aula virtual.

3. El alumno dispone de la Guía Didáctica que le permite abordar el material propuesto.

4. El alumno dispone de un Contrato Pedagógico, instrumento en el que se especifica derechos/obligaciones de alumnos y docentes.

5. Se dispone de un espacio de comunicación que permite al alumno resolver dificultades técnicas.

6. Se dispone de espacios de Interacción alumno/alumno/ docente.

7. Están contempladas todas las componentes de una Unidad Didáctica (UD).

8. El docente cuenta con asesoramiento Pedagógico y Tecnológico permanente.

9. El alumno dispone de información para participaren trabajos colaborativos.

10. Se conoce el grado de cumplimiento de las expectativas del alumno.

Instrumentos de Seguimiento y Evaluación

\begin{tabular}{|l|l|l|}
\hline Indicadores de resultado & Instrumento & Descripción \\
\hline $\begin{array}{l}\text { 1. El aula virtual posee una sección de } \\
\text { Información General, que permite al alumno }\end{array}$ & $\begin{array}{l}\text { Instrumento para verificación de } \\
\text { secciones de un Aula Virtual. }\end{array}$ & $\begin{array}{l}\text { Permite verificarla existencia de } \\
\text { secciones imprescindibles en un }\end{array}$
\end{tabular}
conocer aspectos esenciales para el desarrollo del curso.

2. Existe una sección que facilita al alumno la exploración y adaptación al aula virtual.

3. El alumno dispone de la Guía Didáctica que le permite abordar el material propuesto.

4. El alumno dispone de un Contrato Pedagógico.

5. Se dispone de un espacio de comunicación que permite al alumno resolver dificultades técnicas.

6. Se dispone de espacios de Interacción alumno/alumno/ docente.

7. Están contempladas todas las componentes de la Unidad Didáctica (UD).

8. El docente cuenta con asesoramiento Pedagógico y Tecnológico permanente.

9. El alumno dispone de información para participaren trabajos colaborativos.

10. Se conoce el grado de cumplimiento de las expectativas del alumno. secciones de un Aula Virtual.

AV.
Considera dimensiones para generar una UD.

En el plan de calidad del PE figuran las tareas de cada proceso, sus responsables y los agentes asignados. calidad del PE.

Guía para intervenir en foros.

Instrumento para Seguimiento de Autogestión de Aprendizaje.

Encuesta de satisfacción alumnos.
Contiene recomendaciones de participación.

Permite al alumno sugerir mejoras en el proceso E/ A y al docente realizar reajustes pertinentes.

Instrumento que contiene dimensiones y criterios para conocer el grado de satisfacción de los alumnos.

Reglamentaciones Vigentes

Norma UNE 66181:2012Gestión de la calidad. Calidad de la formación virtual. http://www.aenor.com/

Ordenanza 04/2007-R Creación del Programa de Campus Virtual de la UNSJ y Unidad Ejecutora del Programa.

Ordenanza No 002/10-CS Reglamento Actividades Académicas en la Modalidad a Distancia de la UNSJ.

\section{Seguimiento y medición de los procesos}

El seguimiento y medición de un proceso se realiza a fin de conocer en qué medida los resultados obtenidos se ajustan a los objetivos propuestos. Permite evaluar la capacidad y eficacia de los procesos y contar con datos objetivos para la toma de decisiones orientadas a mejorarlos. La norma ISO 9000:2000 refiere a estos términos: a) Capacidad: Aptitud de una organización, sistema o proceso para realizar un producto que cumple requisitos para ese producto; y b) Eficacia: Extensión en la que se realizan las actividades planificadas y se alcanzan los resultados planificados. 
Además si la organización desea el camino hacia la excelencia atendiendo la norma ISO 9004: 2000 y el enfoque EFQM, deberá demostrar que sus procesos son también eficientes, esto es que optimiza los recursos que utiliza. Para determinar la capacidad, eficacia y eficiencia de un proceso se utilizan los indicadores de Proceso. Algunos autores señalan las siguientes características y requisitos de los indicadores: Deben proporcionar información acerca de algo sobre lo que se desea conocer, establecer valoraciones y permitir tomar decisiones para la mejora de la institución, en su totalidad o en alguna de sus partes. Deben tener relación con los objetivos y con el objeto de evaluación. La identificación de los indicadores debe ser contextualizada y consensuada, deben ser formulados con claridad y deben estar bien fundamentados, a fin de que permitan intervenir para la mejora (Beltrán, 2013 ; González 2011).

\subsection{Instrumentos para evaluar el cumplimiento de indicadores del proceso Ejecución del Curso}

A fin de realizar la evaluación, la institución que pone en marcha el curso debe generar registros y/o instrumentos para evidenciar el funcionamiento de los procesos. A continuación se describirán los instrumentos utilizados para evaluar algunos indicadores definidos para este proceso. Los indicadores 1a 7 ponen énfasis en los procesos de interactividad alumno con el material de aprendizaje y la tecnología. La siguiente tabla 4 muestra parte del instrumento elaborado para la revisión de la unidad didáctica propuesta por el docente:

Tabla 4: Instrumento para revisión de diseño unidad didáctica.

Estimado Docente: Este instrumento ha sido generado a fin que Ud. pueda verificar el cumplimiento de las reglamentaciones vigentes para la edición de una unidad didáctica. Le proponemos marcar con una cruz los ítems considerados en su propuesta.

Objetivos de aprendizaje

¿Están enunciados en las tres dimensiones: conceptual, actitudinal y procedimental?

¿Están contextualizados atendiendo el grupo destinatario?

¿Ha planteado objetivos para resolver los obstáculos epistemológicos de su disciplina?

¿Explicitó la intencionalidad de su propuesta para lograr la reciprocidad del alumno, de modo que pueda gestionar responsablemente su aprendizaje?

Contenidos

¿Realizó el esquema conceptual de la unidad a implementar (contribuye a organizar y determinar el alcance de los contenidos)?

¿La selección de los contenidos es coherente con los objetivos propuestos?

¿La forma de presentación de los contenidos promueve la reflexión, el diálogo, y no son meramente informativos?

¿El lenguaje utilizado para el desarrollo de los contenidos es claro, directo y adecuado al nivel del alumno?

Recursos Didácticos

¿Elaboró una guía que oriente al alumno en el tratamiento de los materiales propuestos?

¿Explicitó la metodología de trabajo?

¿Colocó mensajes de estímulo?

¿Incluyó distintos tipos de actividades?

\begin{tabular}{|l|l|}
\hline Tipo de Actividad & \\
\hline Iniciación-motivación & \\
\hline Explicitación de conocimientos previos & \\
\hline Reestructuración de ideas & \\
\hline Desarrollo o aplicación de nuevas ideas & \\
\hline Relacionadas con el contexto socio-cultural & \\
\hline Revisión & \\
\hline Refuerzo y recuperación & \\
\hline Ampliación & \\
\hline Evaluación & \\
\hline
\end{tabular}

¿Las actividades propuestas están orientadas al cumplimiento de objetivos enunciados?

¿Propone estrategias y actividades para promover autogestión del aprendizaje? ¿Explicita claramente el rol de profesor/tutor y del alumno?

¿Propone estrategias y actividades para promover el aprendizaje colaborativo, basadas en el debate, el intercambio de ideas, análisis crítico y consenso final? ¿Explicita claramente el rol del profesor/tutor y del alumno?

¿Promueve una adecuada y justificada combinación de estrategias presenciales y online?

¿Promueve la utilización de estrategias de aprendizaje basadas en la búsqueda, manejo y gestión de la información?

¿Las imágenes, audio y videos utilizados son coherentes con el contenido textual y colaboran en la construcción del conocimiento?

¿Respeta la normativa y usos correspondientes a derechos de autor y de propiedad intelectual de los materiales utilizados?

Evaluación

¿Planificó el seguimiento del aprendizaje de los alumnos?

¿Ha explicitado los criterios de evaluación?(aspectos a evaluar: actitudinales, conceptuales y procedimentales y tipo de evaluación: cuantitativa, cualitativa)

En la evaluación formativa del alumno, ¿tiene en cuenta sus tareas individuales, grupales, la pertinencia de sus intervenciones en foros y chats, entre otros? 
Los indicadores 6 y 9 refieren a la interacción entre los actores del proceso: alumno/docente y alumnos entre sí. La interacción alumno/docente (indicador 6) permite resolver dificultades de orden disciplinar y/o tecnológicas. El indicador 9 apunta a la posibilidad de realizar trabajo colaborativo, para ello es de fundamental importancia que el alumno conozca aspectos a tener en cuenta para la participación en los foros, tales como rol del profesor y del alumno, modo de intervención, entre otros. El indicador 8 señala la posibilidad del docente de contar con apoyo pedagógico y tecnológico en forma permanente. En el plan de calidad del PE, materializado mediante dotProyect, deben figurar los responsables asignados a tal fin.Los indicadores 1 a 9 están dirigidos a la calidad del servicio, el indicador 10 refiere al otro principio de la Calidad Total orientado a la satisfacción del usuario. Para determinar el cumplimiento de las expectativas del alumno se propone una evaluación permanente y otra evaluación final. En la primera se utiliza el instrumento Seguimiento de la Autogestión del Aprendizaje, de utilidad para alumnos y docentes. Los alumnos pueden tomar conciencia de la autogestión de su aprendizaje y así lograr paulatinamente mayores niveles de autonomía, el docente podrá conocer los temas que presentaron mayor dificultad, a fin de realizar acciones pertinentes. Su formato general se muestra en la tabla 5 siguiente.

Tabla 5: Instrumento de Seguimiento de la Autogestión del Aprendizaje

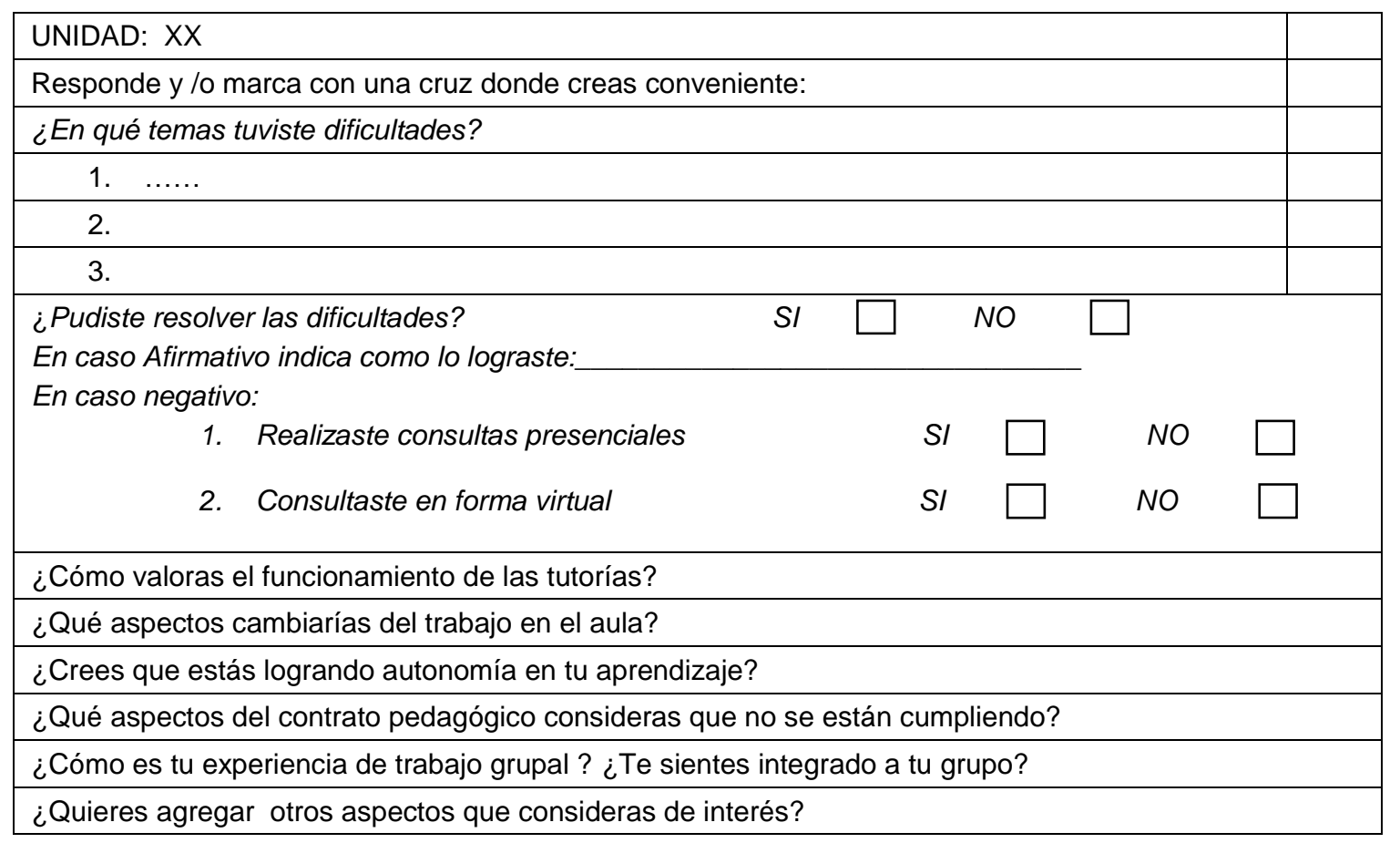

La evaluación final, realizada al concluir el curso, es una encuesta para determinar el grado de satisfacción del alumno, objetivos alcanzados, aspectos destacados a ser potenciados y aspectos a mejorar en próximas ediciones del curso. Esta encuesta ha sido generada según el Modelo de Desarrollo y Utilización de cuestionarios de Satisfacción de Clientes propuesta por Bob Hayes, que permite construir instrumentos de evaluación estadísticamente fiables. Con estos instrumentos se podrán obtener los atributos que son más importantes para los usuarios o de los que están menos satisfechos, así como realizar la reingeniería en los procesos que lo requieran (Llarena, 2008; Llarena et al., 2010). Se consideran en esta evaluación dimensiones relacionadas con la interactividad alumno /material-Aspectos didácticos y Aspectos Curriculares- y la interactividad alumno/recursos tecnológicos -Dimensión Entorno Tecnológico. La dimensión Aspectos Didácticos hace referencia a la funcionalidad y adecuación de los materiales presentados. Entendiendo por materiales a todos los recursos de aprendizaje que se ponen a disposición del alumno en el AV, contenidos, guías didácticas, actividades prácticas, software específico, recursos multimedia, bibliografía, entre otros. La dimensión Aspectos Curriculares considera la profundidad, validez, adecuación y cantidad de contenidos propuestos. La tabla 6 muestra la sección del cuestionario de satisfacción confeccionado, que contiene los estándares considerados para evaluar la interactividad.

Por la importancia que en este proceso educativo tienen los aspectos relacionados con el aprendizaje colaborativo, la interacción alumno/alumno se evalúa a través de su experiencia en las actividades grupales y vía foro. Para evaluar la interacción alumno/tutor se ha considerado el criterio Tutorías en el que se valora profesionalismo, responsabilidad y compromiso del tutor en el cumplimiento de sus tareas, tal como se muestra en la tabla 7. 
Tabla 6: Criterios para evaluar Interactividad

\begin{tabular}{|c|c|c|c|c|c|}
\hline \multicolumn{6}{|c|}{$\begin{array}{l}\text { Hemos concluido esta experiencia de vivenciar la Educación no Presencial como complemento de los procesos de } \\
\text { enseñanza/aprendizaje presenciales. Necesitamos realizar un balance que nos permita identificar los logros y } \\
\text { obstáculos surgidos a lo largo de este proceso Algunos de los enunciados deberán ser evaluados en la escala de } 1 \\
\text { (puntaje mínimo) a } 5 \text { (máximo puntaje). Si el puntaje es } 3 \text { o inferior, le solicitamos indicar los aspectos que Ud. } \\
\text { considera deben ser mejorados En otros casos deberá seleccionar entre varias opciones, marcando con una cruz la } \\
\text { que considere corresponde a lo experimentado durante el curso. Si la opción seleccionada es Generalmente, A veces } \\
\text { o Nunca, por favor especifique sus dificultades o disconformidades. Su opinión representa un valioso aporte que } \\
\text { enriquecerá la experiencia. Su sinceridad será muy apreciada. Gracias }\end{array}$} \\
\hline Aspectos curriculares & 1 & 2 & 3 & 4 & 5 \\
\hline \multicolumn{6}{|c|}{ Adecuación de contenidos para la comprensión de la temática. } \\
\hline \multicolumn{6}{|c|}{ Nivel de profundización de contenidos. } \\
\hline \multicolumn{6}{|c|}{ Extensión de contenidos para la comprensión de las temáticas. } \\
\hline \multicolumn{6}{|l|}{ Claridad en la presentación de contenidos. } \\
\hline Propuesta didáctica & 1 & 2 & 3 & 4 & 5 \\
\hline \multicolumn{6}{|c|}{ Funcionalidad de documentos para la comprensión del tema. } \\
\hline \multicolumn{6}{|l|}{ Adecuación de cantidad de material de estudio propuesto. } \\
\hline \multicolumn{6}{|c|}{ Coherencia entre actividades planteadas y material presentado. } \\
\hline \multicolumn{6}{|c|}{ Coherencia de evaluación propuesta y actividades realizadas. } \\
\hline \multicolumn{6}{|c|}{ Eficacia de trabajo colaborativo (foros y actividades grupales). } \\
\hline Entorno tecnológico & 1 & 2 & 3 & 4 & 5 \\
\hline \multicolumn{6}{|l|}{ Facilidad para acceder a los distintos materiales. } \\
\hline Facilidad para Instalación y uso del Software utilizado & & & & & \\
\hline
\end{tabular}

Tabla 7: Criterios para evaluar Interacción con Tutor

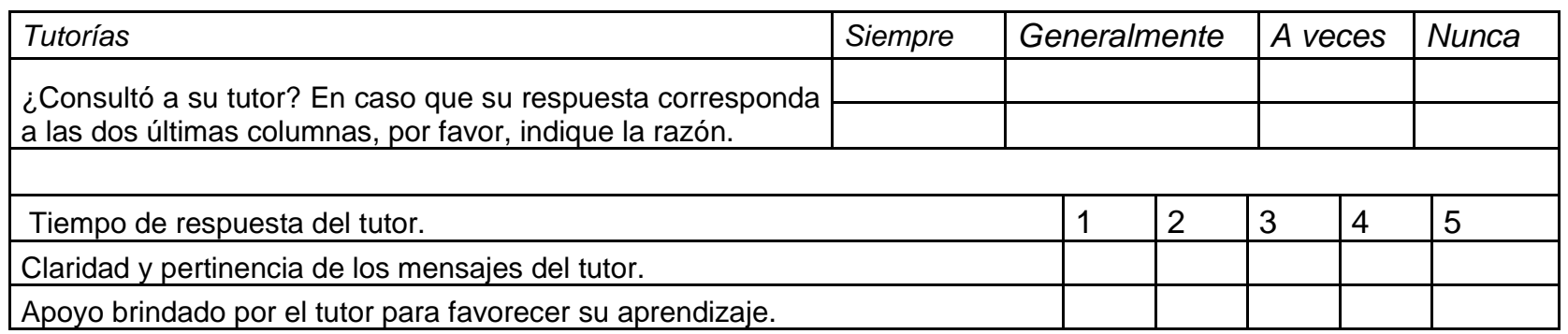

Para determinar la fiabilidad de estos cuestionarios, esto es el grado de coincidencia entre los valores obtenidos a partir de los cuestionarios y la verdadera opinión de los usuarios, se han desarrollado diferentes enfoques y métodos (Hayes, 1993). De los métodos para medir la fiabilidad, propuestos en el marco de la Teoría Clásica de los Tests, el de consistencia interna es el más utilizado. Este método analiza la relación entre las diferentes partes de un mismo instrumento (Ledesma et al, 2002), y utiliza el coeficiente alfa de Cronbach para valorar la consistencia del cuestionario completo o consistencia global. (Cronbach, 1973; Llarena, 2008). El grado de fiabilidad exigido, reflejado en el valor de este coeficiente, varía en función del tipo de estudio. En los estudios exploratorios, se exige que alcance un valor de 0.6 , mientras que en el resto de los estudios se considera una escala fiable si el alfa de Cronbach es superior a 0.7. (Hayes, 1993).

\section{CONCLUSIONES}

La implementación de un SGC para la gestión y puesta en marcha de cursos en la modalidad no presencial, permite a las instituciones educativas contar con un plan de calidad que especifica claramente como se planifica, administra, desarrolla, evalúa y mejora un Proyecto Pedagógico.

La definición de los procesos con un nivel de especificidad adecuado, facilita el desarrollo óptimo de todas y cada una de las actividades involucradas en ellos, así como la identificación de los actores intervinientes, sus roles y responsabilidades.

Disponer de instrumentos de evaluación que atiendan estándares contextualizados pero que a la vez se ajusten a los aceptados internacionalmente, encamina a la institución hacia la idoneidad requerida en el contexto de la globalización y a su vez faculta a los usuarios alumnos a la comparación y evaluación de distintas propuestas educativas, a fin de seleccionar aquella que se adecue a sus necesidades, intereses y expectativas. 


\section{REFERENCIAS}

Aja, L. Gestión de información, gestión del conocimiento y gestión de la calidad en las organizaciones. ACIMED v.10 n.5 2002 Ciudad de La Habana versión impresa ISSN 1024-9435

Beltrán, J., M. Carmona, R. Carrazco, M. Rivas y M. Tejedor (Instituto Andaluz de Tecnología), Guía para una Gestión basada en procesos (2009) ,(en línea), ISBN 84-923464-7-7, http://excelencia.iat.es/files/2012/08/2009.Gestión-basada-procesos-completa.pdf. Acceso 17 de abril (2013) Cronbach, L., On estimates of test reliability. The Journal of Educational Psychology, 34 (4), 485-494 (1973). Crosby, P., Calidad para el Siglo XXI, pp 105, McGraw Hill, Bogotá, Colombia (1998).

De Marco T. Controlling Software Proyects. Prentice Hall, New York (1986)

Deming, W.E. Calidad, Productividad y Competitividad. La salida de la crisis. ISBN 84-87189-22-9. Madrid. Díaz de Santos. S.A (1986).

Díaz, M. Estándares mínimos de calidad para la creación y funcionamiento de programas universitarios de pregrado. Instituto Colombiano para el Fomento de la Educación Superior, Bogotá. (2001).

Feigenbaum, A. Total Quality Control McGraw-Hill ISBN: 0070220034 (2012)

González, L. Conceptos Fundamentales de la Excelencia Modelo EFQM de Excelencia Módulo V. Curso de Formación en Evaluación y Acreditación de la Educación Superior. http://www.lineaverdemunicipal.com/ Guias-buenas-practicas-ambientales/es/e-Modelo-EFQM.pdf Acceso15 de mayo de 2013. (2011)

Hayes, B., Measuring Customer Satisfaction ASQ Quality Pres, Mistwakee Wisconsin, USA (1992). Juran, J. M. y Gryna F.M, Análisis y planificación de la calidad, pp 55, McGraw Hill, México (1995). Juran, J. M. y Gryna F.M, Manual de Control de Calidad. Cuarta edición. España, McGrawHill/Interamericana. (1993).

Hernández G., Arcos J., Sevilla J. Gestión de la calidad bajo la Norma ISO 9001 en instituciones públicas de educación superior en México. Revista Calidad en la educación, 39, 82-115 (2013)

ISO 9000:2000, International Organization for Standardization. Sistemas de gestión de la calidad. Fundamentos y vocabulario, Suiza (2005)

ISO 9001:2008 International Organization for Standardization. Sistemas de gestión de la calidad Requisitos. Suiza. (en línea) http://www.iso.org/iso/home. Acceso10 de junio de 2013. (2008)

Ledesma, R, G.Molina y P. Valero, Análisis de consistencia interna mediante Alfa de Cronbach: un programa basado en gráficos dinámicos (en línea), Acceso Noviembre de 2013, http://pepsic.bvspsi.org.br/pdf/psicousf/ v7n2a03.pdf (2002).

LLarena, M, Metodología para la Evaluación de la Calidad de Estrategias Didácticas de Cursos a Distancia (MACCAD). Form. Univ., vol.1, no.2, p.37-46. ISSN 0718- (2008).

LLarena, M, S. Villodre y A. Cattapan, Calidad en educación no presencial. Metodología de seguimiento y evaluación de cursos, 1ํe edición,5-154, ISBN: 978-3-659-01235-8, Editorial Académica Española, Berlín, Alemania ( 2012).

Moneta, A. y S. Soria, Indicadores de calidad para la gestión docente en entornos virtuales de enseñanza y aprendizaje (EVEA). Hacia un modelo de selección, III Jornadas sobre Experiencias e Investigación en Educación a Distancia y Tecnología Educativa-PROED-UNC. Córdoba, Argentina, (2013)

Ronald,Y.y L.Rodríguez, Manual de DotProject. Rol Administrador (2007), http://www.buenastareas.com/ensayos/Manual-Dotproject/3639243.html. Acceso 10 de noviembre (2013).

Rubio, M y equipo de expertos. Memoria del Proyecto "Centro Virtual para el Desarrollo de Estándares de Calidad para la Educación Superior a Distancia en América Latina y el Caribe" CALED (Instituto Latinoamericano y del Caribe de Calidad en Educación Superior a Distancia (2010). http://educ-al.org/caled/ Acceso 29 de mayo de 2014.

Tiana, A. Curso online de Formación en Evaluación y Acreditación de la Educación Superior Unidad 10: Criterios de Evaluación e Indicadores. fm 6. Disponible en hhtp://www.iesalc.unesco.org.ve/cursos/Formación Evaluación. Acceso15 de mayo de 2013. (2010).

UNE 66181:2012, Gestión de la calidad. Calidad de la formación virtual, AENOR, España (2012) 
